\title{
3D Magneto-Buoyancy-Thermocapillary Convection of CNT-Water Nanofluid in the Presence of a Magnetic Field
}

\author{
Lioua Kolsi ${ }^{1, *(\mathbb{D})}$, Salem Algarni ${ }^{2}$, Hussein A. Mohammed ${ }^{3, *(1)}$, Walid Hassen ${ }^{4}$, \\ Emtinene Lajnef ${ }^{4}$, Walid Aich ${ }^{1,5}$ and Mohammed A. Almeshaal ${ }^{6}$ \\ 1 Department of Mechanical Engineering, College of Engineering, Ha'il University, Ha'il City 2240, \\ Saudi Arabia; w.aich@uoh.edu.sa \\ 2 College of Engineering, Mechanical Engineering Department, King Khalid University, Abha 61421, \\ Saudi Arabia; saalgarni@kku.edu.sa \\ 3 School of Engineering, Edith Cowan University, 270 Joondalup Drive, Joondalup, WA 6027, Australia \\ 4 Laboratory of Metrology and Energy systems, Monastir, University of Monastir, Monastir 5000, Tunisia; \\ hassen.walid@gmail.com (W.H.); laj.emtinene_nasa@yahoo.fr (E.L.) \\ 5 Materials, Energy and Renewable Energies Research Unit, Faculty of Sciences, University of Gafsa, \\ Gafsa 2112, Tunisia \\ 6 Department of Mechanical Engineering, College of Engineering, Al Imam Mohammad Ibn Saud Islamic \\ University, Riyadh 11432, Saudi Arabia; maalmeshaal@imamu.edu.sa \\ * Correspondence: 1.kolsi@uoh.edu.sa (L.K.); Hussein.mohammed@ecu.edu.au or \\ Hussein.dash@yahoo.com (H.A.M)
}

Received: 16 December 2019; Accepted: 19 February 2020; Published: 25 February 2020

\begin{abstract}
A numerical study is performed to investigate the effects of adding Carbon Nano Tube (CNT) and applying a magnetic field in two directions (vertical and horizontal) on the 3D-thermo-capillary natural convection. The cavity is differentially heated with a free upper surface. Governing equations are solved using the finite volume method. Results are presented in term of flow structure, temperature field and rate of heat transfer. In fact, results revealed that the flow structure and heat transfer rate are considerably affected by the magnitude and the direction of the magnetic field, the presence of thermocapillary forces and by increasing nanoparticles volume fraction. In opposition, the increase of the magnetic field magnitude leads to the control the flow causing flow stabilization by merging vortexes and reducing heat transfer rate.
\end{abstract}

Keywords: buoyancy thermocapillary convection; magnetic field direction; CNT-water nanofluid; Marangoni number; flow control.

\section{Introduction}

Over the last few decades, there has been growing interests on thermocapillary-buoyancy convection since it occurs in several important applications such as crystal growth, welding and coating. Nevertheless, the intriguing behavior and instabilities encountered in this type of flow exhibit a challenge to overcome. Therefore, a sizable amount of studies focused on buoyant thermocapillary convection instabilities. Gillon and Homsy [1] carried out an experimental study on thermocapillary-buoyancy convection of silicone oil. They used the PIV (Particle Image Velocimetry) technique to evaluate the effect of capillary forces on flow structure.

Kanouff and Grieif [2] studied thermocapillary convection oscillations of liquid metals in a square cavity. They mentioned that, for low Marangoni number the flow structure is characterized by two symmetrical vortices. Nevertheless, for a larger value of Marangoni of the order of 1200, the flow 
becomes unstable and there are oscillations caused by the surface tension. This bifurcation gives birth to two opposing motion vortices. Similarly, Chen and Hwu [3] showed that there is a critical Marangoni number above which the thermocapillary flow undergoes an oscillatory movement with a frequency of oscillation while the flow is stable for lower values. This critical number of Marangoni that is responsible for oscillation increases with the increase of Biot number. Semma et al. [4] investigated the effects of thermocapillary convection on fluid phase instabilities and the horizontally directed solid-liquid interaction of a low Prandtl fluid. They studied the transitions in a melt bath in the presence of thermocapillary convection and the interaction with the solidification front. This work shows that the solid-liquid interface is largely affected when the surface forces dominate over the volume forces. However, the presence of thermocapillary convection improves the stability of the flow and delays transitions to the oscillatory regime. Behniat et al. [5] conducted a numerical study that combines buoyancy convection and thermocapillary convection in a three-dimensional cubic enclosure with material free surface having a Prandtl number equal to 7. Authors discuss the effects of Marangoni number sign variation on three-dimensional convection for different Rayleigh number values. This study proved that for positive values of $\mathrm{Ma}$, the flow structure is monocellular. For higher values of Ma, the flow becomes more intense. They mention that for positive values of Marangoni, thermocapillary convection is not so remarkable, whereas for negative values of Marangoni the flow structure is more interesting and complex. In this case, the authors confirmed that there is an opposition between the effects of buoyancy convection and the effect of Marangoni. Babu and Korppela [6] compared the 2D and 3D thermocapillary convection flows and found that for low Ma the behavior is similar. This statement is in concordance with that of Zebib et al. [7]. Similarly, Ramanan and Korpela [8] mentioned that the velocity of the free surface is greater for the three-dimensional case than the two-dimensional one for high Ma, while this is not the case when Ma is low. They also reported that for low values of Marangoni number, conduction dominates convection and consequently the temperature is decreasing linearly all along the free surface. However, for larger Ma, convection dominates, and the temperature gradient is wider near the cold wall.

Therefore, based on the abovementioned studies, it is necessary to have a process to delay transitions to an oscillatory unsteady state. In this context, many analytical, experimental and numerical studies have shown that the use of an external magnetic field can control the flow and the heat transfer. Morthland and Walker [9] studied the effect of a horizontal magnetic field on thermocapillary convection. They mentioned that due to the generated Lorentz force, the thermocapillary convection is reduced and becomes localized near the free surface. Rudraiah et al. [10] carried out a study that shows the effects of magnetic fields parallel to gravity on a flow due to combined buoyancy convection and thermocapillary convection of a metal liquid. They noticed that the heat transfer is enhanced by increasing Ma and decreases with Ha. Jiang et al. [11] investigated coupled forced-thermocapillary convection of Nanofluid flow induced by bubble in minichannel. They focused on the effects of nanoparticle concentration and diameter on thermal transport and they have found that the local heat transfer can be controlled by adjusting the nanoparticles' size and concentration. They also mentioned that the thermocapillary jet in minichannel can strongly improve the thermal energy transport near gas bubble. In another work, Jiang and Zhou [12] reported a computational analysis of heat transfer and entropy generation driven by nanofluids thermocapillary convection around a bubble in a cavity. They concentrated on the influence of nanoparticles diameter and volumetric fraction on flow behavior, heat transfer achievement and entropy creation. The obtained results reveal that the increase of nanoparticles volume fraction enhances the heat transfer rate even the convective intensity is decreased, and the local entropy generation is reduced. Nevertheless, opposite results are observed with larger nanoparticles diameter. Buoyancy-thermocapillary driven convection flow and heat transfer inside 3D differentially heated cavity filled with $\mathrm{Al}_{2} \mathrm{O}_{3}$ nanofluid are carried out by Kolsi et al. [13]. It was mentioned that the increase of nanoparticles volume fraction leads to an enhancement of heat transfer as well as the flow intensity regardless the Marangoni number values. Aminfar et al. [14] conducted a numerical investigation of thermocapillary-buoyancy driven convection 
of nanofluids in a floating zone. In this study, spherical shape $\mathrm{Al}_{2} \mathrm{O}_{3}$ nanoparticles are added to silicone oil as a base fluid. They indicated that, in the absence of gravity effect, the increase of nanoparticles concentration leads to the deterioration of thermocapillary convective characteristics. Even in the presence of gravity, adding $\mathrm{Al}_{2} \mathrm{O}_{3}$ reduces the thermocapillary and buoyancy driven nanofluid Nusselt number. Abdullah et al. [15] undertake the Marangoni convection stability analysis in a horizontal nanofluid layer cooled from the top boundary and heated from below. They reported that the steady state solution across the layer is well approximated by an exponential distribution of nanoparticle volume fraction and a linear distribution of temperature. Moreover, a new behavior is pointed out which consequently leads to considerably different stability boundaries from previous predictions. Jiang and Zhou [16] analyzed the flow and heat transfer characteristics of nanofluids thermocapillary driven convection in a rectangular cavity with two types of nanofluids (water- $\mathrm{Al}_{2} \mathrm{O}_{3}$ and $\mathrm{PGW}-\mathrm{ZnO}$ ). They observed a nonmonotonic variation of convection intensity and heat transfer rate with the increasing of nanoparticles in the case of water- $\mathrm{Al}_{2} \mathrm{O}_{3}$ whereas, in the case of PGW-ZnO, there is a monotonous decrease. Numerical investigation of combined buoyancy-Marangoni convection heat and mass transfer of power-law nanofluids was conducted by Zhuang and Zhu [17]. They reported that, when the buoyancy force is intensified, the influence of the surface tension on the strength of heat and mass transfer becomes negligible. Moreover, an increase in the average Nusselt and Sherwood numbers was observed with the increase of the Marangoni and thermal Rayleigh numbers due to the combined effects of buoyancy-thermocapillary convection. Lee et al. [18] studied, experimentally and theoretically, the combined thermocapillary and natural convection in rectangular containers with localized heating. The flow changes from bidimensionnal to three-dimensional oscillatory structure with periodic spanwise variation. Furthermore, the flow follows weak and strong periods in one cycle of oscillation for which the highest level occurs along the interface between the main flow cell and the stagnant region below it. Eventually, it should be emphasized that a sizable amount of other related studies can be found in the literature review [19-25].

As described in the above literature review the thermocapillary convection [1-10] and Magnetohydrodynamic (MHD) convection [26,27] were widely studied. A recent published paper by Al-Rashed et al. [28] who studied the effect of an inclined magnetic field on CNT-nanofluid natural convection. In our study a similar configuration based on from the work of the Al-Rashed et al. [28] is investigated and the novelty is the consideration of additional effect of surface tension which its intensity is characterized by Marangoni number (Ma). Except the Marangoni number all the chosen ranges of the parameters governing the phenomenon were inspired from the work of the Al-Rashed et al. [28]. Thus, the subject of the current paper is the effect of the direction of a magnetic field on CNT-water nanofluid thermocapillary natural convection in a cubic cavity.

\section{Problem Statement}

The studied configuration is illustrated on Figure 1; a 3D differentially heated cavity filled with CNT-water nanofluid is considered. The upper surface is free, and an inclined magnetic field is imposed. Table 1 presents the thermophysical properties of the base fluid (water) and CNT.

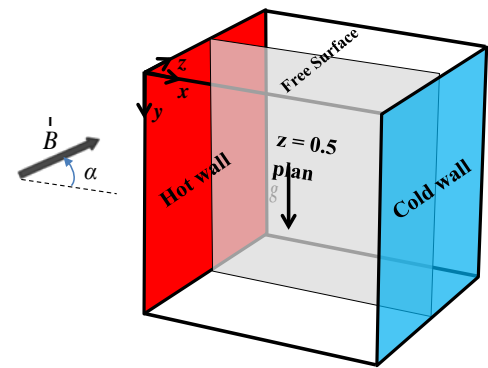

(a)

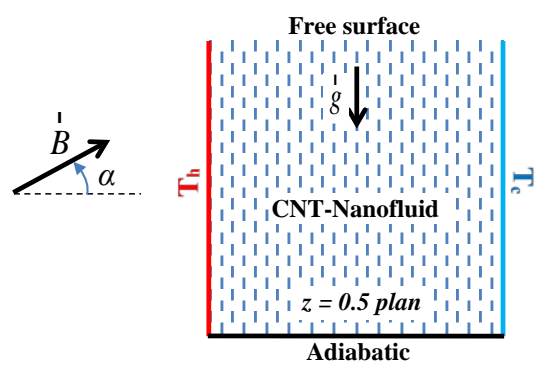

(b)

Figure 1. (a): Considered configuration with coordinates and (b) the central plan $(z=0.5)$. 
Table 1. Properties of CNT (Carbon Nanotube) and water [28].

\begin{tabular}{ccc}
\hline Properties & $\mathbf{C N T}$ & $\mathbf{H}_{\mathbf{2}} \mathbf{O}$ \\
\hline$C_{p}\left(\mathrm{~J} \mathrm{~kg}^{-1} \mathrm{~K}^{-1}\right)$ & 425 & 4179 \\
$\rho\left(\mathrm{kg} \mathrm{m}^{-3}\right)$ & 2600 & 997.2 \\
$k\left(\mathrm{~W} \mathrm{~m} \mathrm{~K}^{-1} \mathrm{~K}^{-1}\right)$ & 6600 & 0.613 \\
$\beta\left(\mathrm{K}^{-1}\right)$ & $1.6 \times 10^{-6}$ & $21 \times 10^{-5}$ \\
$\sigma_{e}\left(\Omega^{-1} \cdot \mathrm{m}^{-1}\right)$ & $4.8 \times 10^{-7}$ & 0.05 \\
\hline
\end{tabular}

\subsection{Governing Equations}

The time depending governing equations are developed via the potential vector velocity-vorticity formalism in a 3D configuration. This formalism eliminates the pressure gradient terms, to simplify the expressions and facilitate the convergence.

The vorticity and the potential velocity vector are:

$$
\overrightarrow{w^{\prime}}=\vec{\nabla} \times \overrightarrow{U^{\prime}}
$$

and

$$
\overrightarrow{U^{\prime}}=\vec{\nabla} \times \overrightarrow{\Psi^{\prime}}
$$

The governing equations are as follows:

$$
\begin{gathered}
-\vec{w}=\nabla^{2} \vec{\Psi} \\
\frac{\partial \vec{w}}{\partial t}+(\vec{U} \cdot \nabla) \vec{w}=(\vec{w} \cdot \nabla) \vec{U}+\frac{v_{n f}}{v_{f}} \operatorname{Pr} \cdot \nabla^{2} \vec{w}-\frac{\beta_{n f}}{\beta_{f}} \operatorname{Ra} \operatorname{Pr} \nabla \times \theta \vec{g}+\frac{\rho_{f}}{\rho_{n f}} \cdot \frac{\sigma_{n f}}{\sigma_{f}} H a^{2} \cdot \operatorname{Pr} \cdot \nabla \times\left(\vec{j} \times \vec{e}_{B}\right) \\
\frac{\partial T}{\partial t}+\vec{U} \cdot \nabla T=\frac{\alpha_{n f}}{\alpha_{f}} \nabla^{2} \theta \\
\vec{j}=-\vec{\nabla} \Phi+\vec{U} \times \vec{e}_{B} \\
\vec{\nabla}^{2} \Phi=\vec{\nabla} \cdot(\vec{U} \times \vec{B})=-\vec{e}_{B} \cdot \vec{w}
\end{gathered}
$$

With

$$
\vec{e}_{B}=\cos (\alpha) \cdot \vec{e}_{x}+\sin (\alpha) \cdot \vec{e}_{y}
$$

With:

$$
\operatorname{Pr}=\frac{v_{f}}{\alpha_{f}}, R a=\frac{g \cdot \beta_{f} \cdot \Delta T \cdot l^{3}}{v_{f} \cdot \alpha_{f}}, \text { and } H a=B_{0} L \sqrt{\frac{\sigma_{f}}{\rho_{f} v_{f}}}
$$

To obtain the abovementioned Equations (4)-(7), the variables: $t^{\prime}, \overrightarrow{U^{\prime}}, \overrightarrow{\psi^{\prime}}, \overrightarrow{\omega^{\prime}}, \overrightarrow{J^{\prime}}, \overrightarrow{B^{\prime}}$ and $\Phi^{\prime}$ are divided by: $l^{2} / \alpha, \alpha / l, \alpha, l^{2} / \alpha, \sigma \cdot v_{0} \cdot B_{0}$ and $l \cdot v_{0} \cdot B_{0}$ respectively.

$$
\theta=\left(T^{\prime}-T_{c}^{\prime}\right) /\left(T_{h^{\prime}}-T_{c}^{\prime}\right)
$$

The surface tension law at the upper free surface is:

$$
\sigma=\sigma_{0}-\gamma\left(T-T_{0}\right)
$$

where $\sigma_{0}$ is the surface tension at the initial temperature $\left(T_{0}\right)$ and $\gamma=-\frac{\partial \sigma}{\partial T}$ is the thermal coefficients of surface tension.

The thermophysical properties of the nanofluid are evaluated as follow: 
Effective density:

$$
\rho_{n f}=\varphi \rho_{n}+(1-\varphi) \rho_{f}
$$

Specific heat:

$$
\left(\rho C_{p}\right)_{n f}=\varphi\left(\rho C_{p}\right)_{n}+(1-\varphi)\left(\rho C_{p}\right)_{f}
$$

Effective thermal conductivity [29]:

$$
\frac{k_{n f}}{k_{f}}=\frac{1-\varphi+2 \varphi \frac{k_{n}}{k_{n}-k_{f}} \ln \left(\frac{k_{n}+k_{f}}{2 k_{f}}\right)}{1-\varphi+2 \varphi \frac{k_{f}}{k_{n}-k_{f}} \ln \left(\frac{k_{n}+k_{f}}{2 k_{f}}\right)}
$$

Effective dynamic viscosity:

$$
\mu_{n f}=\frac{\mu_{f}}{(1-\varphi)^{2.5}}
$$

Electrical conductivity [26]:

$$
\sigma_{e n f}=\sigma_{f}\left[1+\frac{3 \cdot \phi \cdot\left(\frac{\sigma_{n}}{\sigma_{f}}-1\right)}{\left(\frac{\sigma_{n}}{\sigma_{f}}+2\right)-\phi \cdot\left(\frac{\sigma_{n}}{\sigma_{f}}-1\right)}\right]
$$

It is to be mentioned that the CNT was used because it has a higher thermal conductivity allowing a better enhancement of heat transfer and its better thermal dispersion and stability in a base fluid (water) compared to other kinds of nanoparticles [30].

The local and average Nusselt numbers are:

$$
N u=\left.\left(\frac{k_{n f}}{k_{f}}\right) \frac{\partial T}{\partial x}\right|_{x=0,1} \text { and } N u_{a v}=\int_{0}^{1} \int_{0}^{1} N u \cdot d y \cdot d z
$$

\subsection{Boundary Conditions}

The boundary conditions used to solve the computational domain are:

- Temperature:

$$
\begin{gathered}
\theta=1 \text { at } x=1 \text { and } \theta=0 \text { at } x=0 \\
\frac{\partial \theta}{\partial n}=0 \text { on adiabatic walls }
\end{gathered}
$$

- Vorticity:

$$
\begin{aligned}
& w_{x}=0, w_{y}=-\frac{\partial U_{z}}{\partial x}, w_{z}=\frac{\partial U_{y}}{\partial x} \text { at } x=0 \text { and } 1 \\
& w_{x}=\frac{\partial U_{z}}{\partial y}, w_{y}=0, w_{z}=-\frac{\partial U_{x}}{\partial y} \text { at } y=0 \text { and } 1 \\
& w_{x}=-\frac{\partial U_{y}}{\partial z}, w_{y}=\frac{\partial U_{x}}{\partial z}, w_{z}=0 \text { at } z=0 \text { and } 1
\end{aligned}
$$

- Potential vector:

$$
\begin{aligned}
& \frac{\partial \Psi_{x}}{\partial x}=\Psi_{y}=\Psi_{z}=0 \text { at } x=0 \text { and } 1 \\
& \Psi_{x}=\frac{\partial \Psi_{y}}{\partial y}=\Psi_{z}=0 \text { at } y=0 \text { and } 1
\end{aligned}
$$




$$
\Psi_{x}=\Psi_{y}=\frac{\partial \Psi_{z}}{\partial z}=0 \text { at } z=0 \text { and } 1
$$

- Velocity:

$$
\begin{gathered}
U_{x}=U_{y}=U_{z}=0 \mathrm{on} \text { all the walls except the upper surface } \\
U_{y}=0, \frac{\partial U_{x}}{\partial y}=-M a \frac{\partial \theta}{\partial x} \text { and } \frac{\partial U_{y}}{\partial y}=-M a \frac{\partial \theta}{\partial z} \text { at } \mathrm{y}=1 \text { (free surface) }
\end{gathered}
$$

Marangoni number is expressed as:

$$
M a=\left(\frac{\partial \sigma}{\partial T^{\prime}}\right) \frac{\Delta T^{\prime}}{\alpha_{n f} \mu_{n f}}
$$

- Electric potential:

$$
\frac{\partial \phi}{\partial n}=0 \text { on all walls }
$$

- Density of current:

$$
\vec{J} \cdot \vec{n}=0 \text { on all walls }
$$

An in-house numerical code is written using Fortran Language to solve the governing equations. The discretization is performed via the control volume method. Uniform grids are used with additional nodes at the boundaries. The central difference scheme and the fully implicit procedure are used to treat the convective terms and the temporal derivatives, respectively. To facilitate the convergence of the solution of the nonlinear algebraic equations, the successive relaxation is opted.

\section{Grid Independency Test and Code Verification}

A grid independency test was performed as presented in Table 2. The tests were carried out for four grids. The average number was taken as testing parameter. The difference between the grids $81^{3}$ and $91^{3}$ is only $0.292 \%$. Thus the grid $81^{3}$ was retained for all simulations.

Table 2. Grid independency test: $\mathrm{Ra}=10^{5}, \alpha=90^{\circ}, \mathrm{Ha}=50$ and $\varphi=0.05$.

\begin{tabular}{cccc}
\hline Grid Size & $\mathbf{N u}_{\mathbf{a v}}$ & \% Increase & Incremental Increase \\
\hline $60^{3}$ & 6.2213 & - & - \\
$71^{3}$ & 6.308 & 1.393599 & - \\
$81^{3}$ & 6.37589 & 2.48485 & 1.076252 \\
$91^{3}$ & 6.3945 & 2.783984 & 0.291881 \\
\hline
\end{tabular}

Table 3 presents, the verification performed by comparing the calculated values of average Nusselt number with those of Ozoe and Okada [27], for a horizontal magnetic field. The comparison shows a good concordance.

Table 3. Average Nusselt number for $\operatorname{Ra}=10^{6}, \operatorname{Pr}=0.054$, and $\alpha=0^{\circ}$ : comparison with Ozoe and Okada [27].

\begin{tabular}{ccc}
\hline & Ha = 100 & Ha = 200 \\
\hline Present code & 4.432 & 2.851 \\
Ozoe and Okada [27] & 4.457 & 2.917 \\
\hline
\end{tabular}

\section{Results and Discussions}

Three-dimensional computational investigation of Magnetohydrodynamic (MHD) thermocapillary convective flow, based on finite volume method, was performed. A differentially heated cubic cavity 
subject to an external magnetic field and filled with carbon nanotubes-water nanofluid is considered. The obtained results revealed that the combined effects of induced buoyancy forces and generated Lorentz forces affected the flow behavior.

In fact, while Lorentz forces reorganize the fluid's molecular concentrations and retard heat transfer, buoyancy induced forces play an important role in its enhancement. Moreover, the electromagnetic forces intensity hinges on the amplitude and magnetic field direction. Indeed, an increasing of Hartmann number is accompanied with an increase of Lorentz forces strength whereas the increase of Rayleigh number leads to buoyancy forces enhancement. Furthermore, adding CNT nanoparticles to the pure water results in convection intensification by dint to their high thermal conductivity. In this investigation, we focus on the impacts of magnetic field direction, concentration of nanoparticles and Hartmann number values on thermal energy transfer rate together with the fluid flow structure within a cubic enclosure. The single phase model was used in numerical simulations and the main findings are displayed in form of temperature iso-surfaces, flow speed projection, mean Nusselt number and particles' trajectories with a variety of parameters including magnetic field direction $\left(\alpha=0^{\circ}\right.$ and $\alpha$ $\left.=90^{\circ}\right)$, CNT nanoparticles volumetric fractions $(0 \leq \varphi \leq 0.05)$, Hartmann number $(0 \leq \mathrm{Ha} \leq 100)$, Marangoni number $\left(-10^{4} \leq \mathrm{Ma} \leq 10^{4}\right)$ and Rayleigh number $\left(10^{3} \leq \mathrm{Ra} \leq 10^{5}\right)$. The flow structure inside the cubic confined enclosure is depicted by particles' trajectories and velocity vector projection. Figure 2 illustrated the path of some particles for pure water and nanofluid with strong concentration of nanoparticles not subjected to magnetic field, $\mathrm{Ra}=10^{5}$ and different Marangoni number values. Obviously, the convection currents are thermally dominated due to the absence of magnetic force and particles rotate in clockwise spirals around the center for pure fluid. In addition, the flow structure is composed of two co-rotating eddies adjacent to the active walls which are subsequently fused by the buoyancy forces and the particle trajectories pile up tightly at the cavity center. However, adding CNT nanoparticles to the base fluid leads to the increase of buoyancy forces and consequently the increase of vortices size. The paths of particles moved towards the actives sides and disappeared in the cavity center. Besides, the growing vortex magnitude is plainly noticeable with the flow speed projection at the mid-plane (Figure 3).

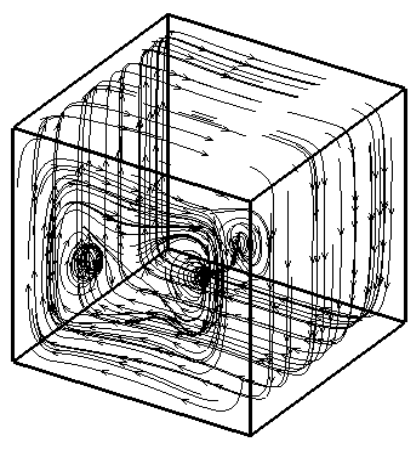

(a) $\mathrm{Ma}=0$

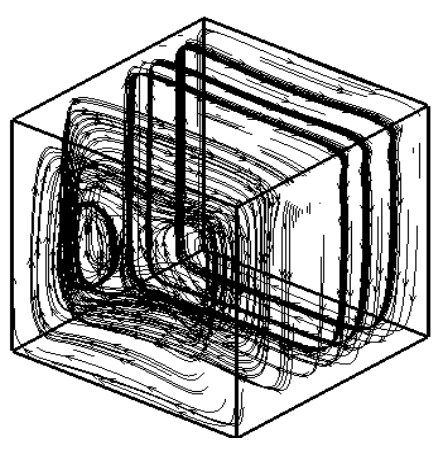

(b) $\mathrm{Ma}=10^{4}$

Figure 2. Some particles' trajectories are not subjected to magnetic field $(\mathrm{Ha}=0)$ for $\mathrm{Ra}=10^{5} ;(\mathbf{a}) \mathrm{Ma}=0$ and (b) $\mathrm{Ma}=10^{4}$.

It can also be noticed that the flow path is dominated by Marangoni effect and adding CNT particles increases the disorder on the flow field trajectory, which makes the central cell that processes this effect become larger (Figure 3). The combined effect of thermocapillary-buoyancy convection causes an increase in the flow intensity, particularly close to the top open surface. In comparison with the case $\mathrm{Ma}=0$, the flow remains bicellular and by dint of thermocapillary-buoyancy forces combination both vortices are relatively larger. For negative Marangoni number values, it should be mentioned that there is penetration of warm nanofluid from the top right corner towards the free 
surface middle owing to the combined influence of the two counter-rotating vortex which results in isotherms distortion in this region.

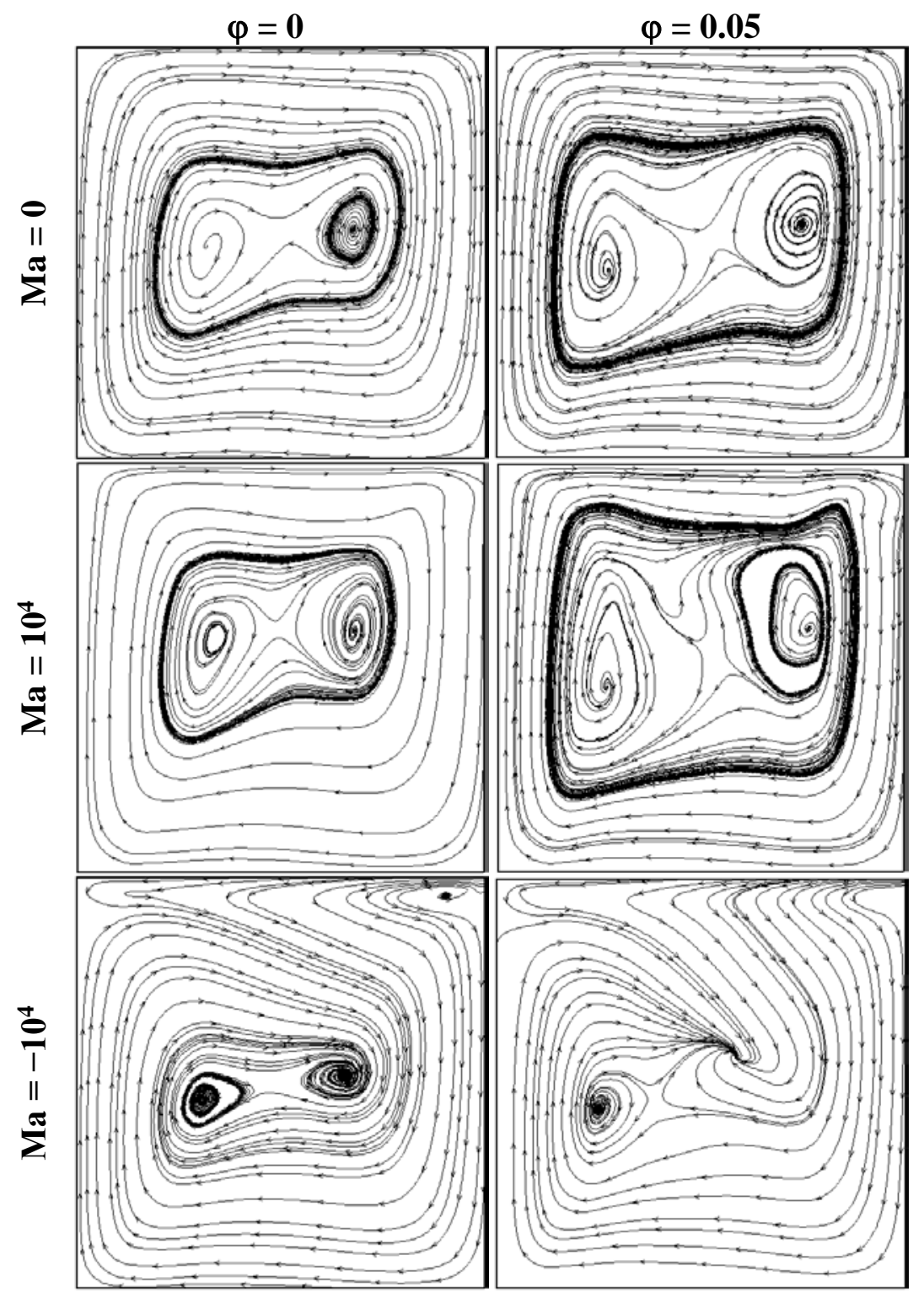

Figure 3. Velocity vectors projection on the central plan for $\mathrm{Ra}=10^{5},(\mathrm{Ha}=0)$ and different Ma.

Figures 4 and 5 illustrate the particles' trajectories at the central plan for different magnetic field directions, $\mathrm{Ha}=100$ and $\mathrm{Ra}=10^{5}$. It was found that the electromagnetic forces eliminate those induced by buoyancy effect. This finding is in concordance with results of Ozoe and Okada [27] who indicated the suppression of the buoyancy force by the induced Lorentz force. Obviously, these forces are orthogonal to each other for $\alpha=0^{\circ}$ (horizontal magnetic field) which leads to the generation of a single vortex at the mid-height of vertical walls. The particles' tracks consist of clockwise recirculation throughout the magnetic field showing an increase in contour level in the direction of the horizontal sides. Furthermore, the electromagnetic forces are removed by buoyancy-induced forces near to the cavity center due to minimization of magnetic induction in this region that leads to a uniform spreading of particles' trajectories. 


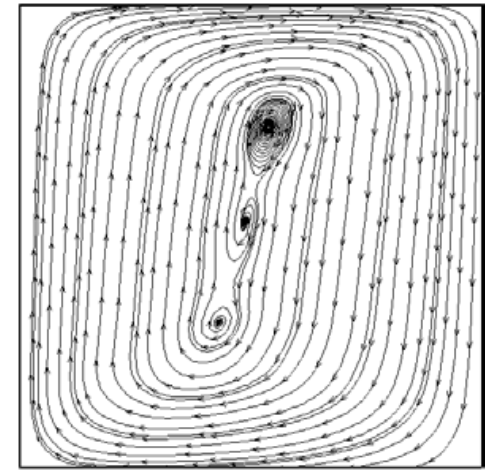

(a) $\alpha=0^{\circ}$ and $\mathrm{Ma}=0$

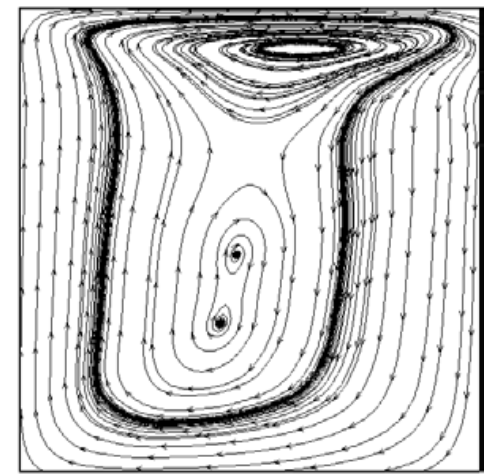

(b) $\alpha=0^{\circ}$ and $\mathrm{Ma}=10^{4}$

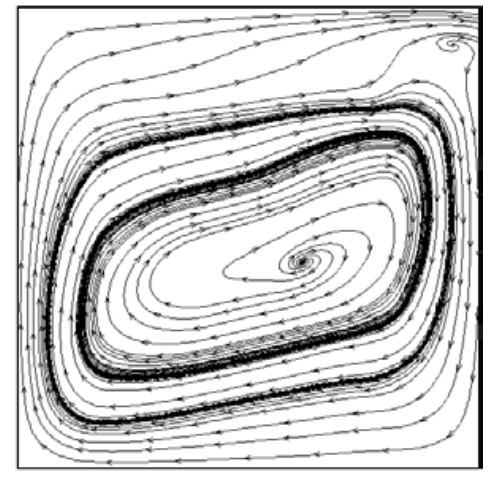

(c) $\alpha=90^{\circ}$ and $\mathrm{Ma}=10^{4}$

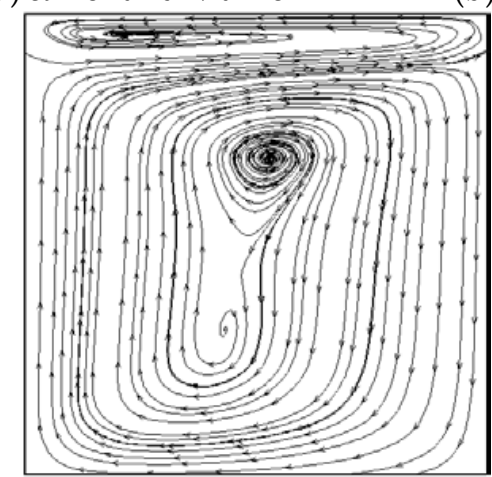

(d) $\alpha=0^{\circ}$ and $\mathrm{Ma}=-10^{4}$

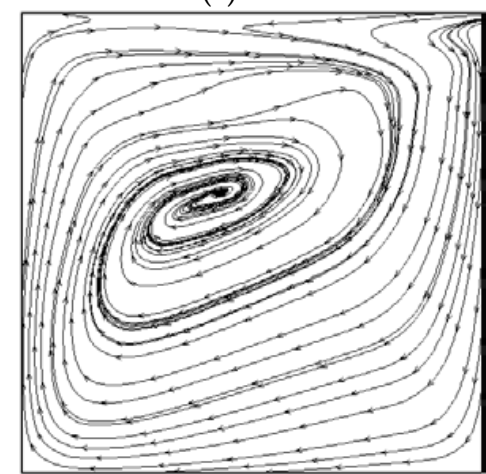

(e) $\alpha=90^{\circ}$ and $\mathrm{Ma}=-10^{4}$

Figure 4. Velocity vectors projection on the central plan for $\mathrm{Ra}=10^{5}, \mathrm{Ha}=100$ and $\varphi=0.05:(\mathbf{a}) \alpha=0^{\circ}$ and $\mathrm{Ma}=0,(\mathbf{b}) \alpha=0^{\circ}$ and $\mathrm{Ma}=10^{4},(\mathbf{c}) \alpha=90^{\circ}$ and $\mathrm{Ma}=10^{4},(\mathbf{d}) \alpha=0^{\circ}$ and $\mathrm{Ma}=-10^{4}$ and $(\mathbf{e}) \alpha=90^{\circ}$ and $\mathrm{Ma}=-10^{4}$.

For an inclination angle of $90^{\circ}$ (Vertical magnetic field), the flow structure consists of two co-rotating swirls with uniform distribution of particles' trajectories across the cavity. The Lorentz forces are found to be enhanced leading to an extension of the magnetic effect to the middle. Owing to the suppression effect of the magnetic field and when its tilt angle reaches $90^{\circ}$, the gravitational and Lorentz forces become parallel and bicellular flow recurs. In addition, increasing the concentration of nanoparticles results in reduction of particles paths contour level, which are compact close to the center.

The appearance of the thermocapillary effect near the free surface of the enclosure is always noted, whether for the positive or negative values of Ma as indicated by Behniat et al. [5]. Furthermore, when the magnetic field strength is increased $(\mathrm{Ha}=100)$, the buoyancy effect is postponed due to the intensification of the Lorenz forces. For an inclination angle of $0^{\circ}$, the decrease in intensity leads to the switch of vortices rotation to vertical direction with an expansion of the spiral waves towards the cavity center. For $\alpha=90^{\circ}$, the strength of buoyancy forces increased leading to a horizontal inclination of vortices. The trajectories of the particles are dense around the center.

For the Marangoni effect on the flow field, it is noted that for $\mathrm{Ma}=10^{4}$ and for an inclination angle $\alpha=90^{\circ}$, the flow is depicted by a single vortex rotating in a horizontal direction relative to gravitational forces. The same thing was observed for $\mathrm{Ma}=-10^{4}$ with a higher intensity at the center of the vortex. Therefore, it can be concluded that if one aims to incline the direction of the magnetic field it can affect not only the buoyancy forces but also the thermocapillarity. 


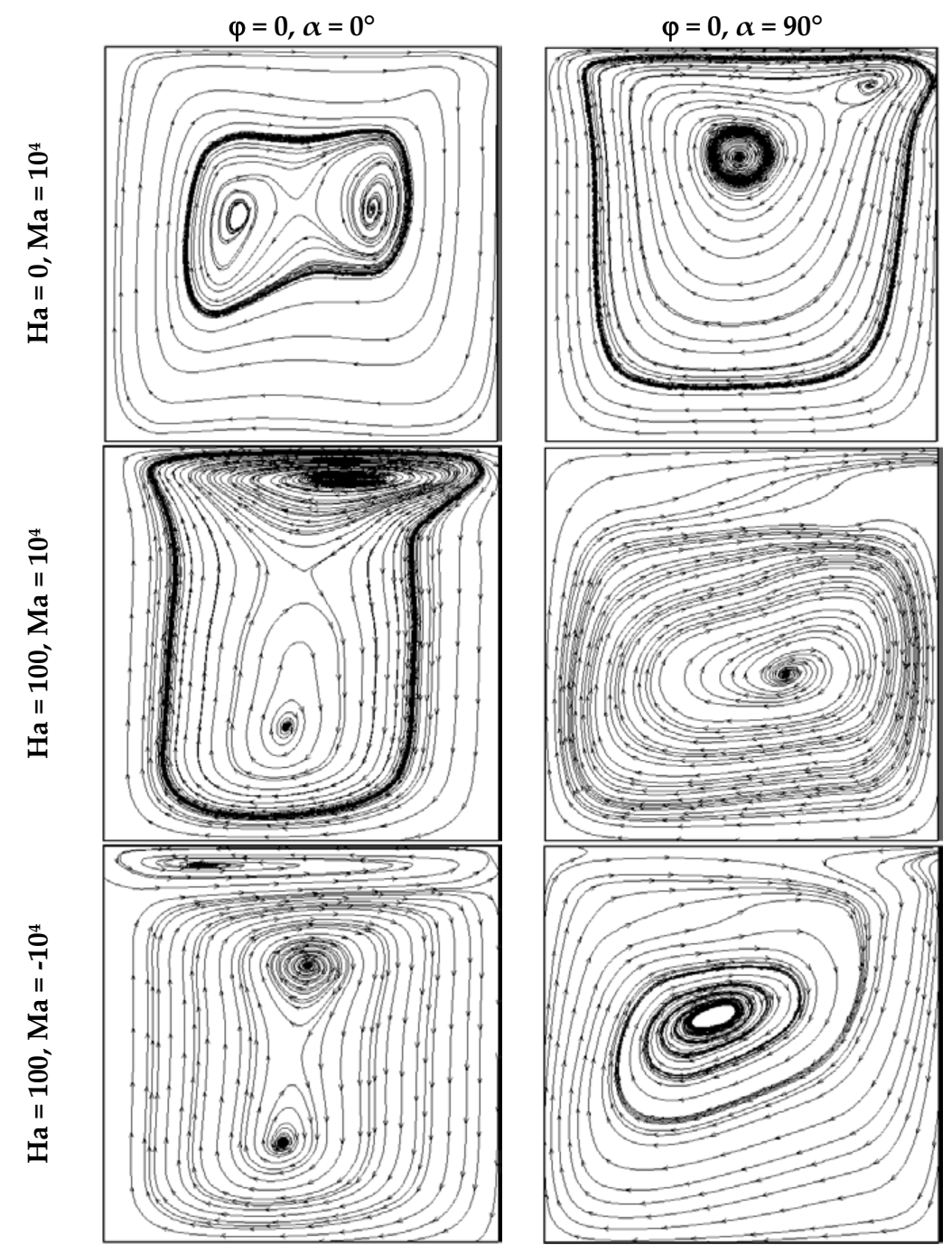

Figure 5. Velocity vectors projection on the central plan for $\operatorname{Ra}=10^{5}$ and $\varphi=0$.

Furthermore, for an inclination angle of $90^{\circ}$, the thermal energy transfer is improved along with an extension of maximum contour levels towards the right upper side. Moreover, under the magnetic effect, there is an attraction of CNT nanoparticles to the left side due to the buoyancy repulsion. Further addition of nanoparticles improves buoyancy and causes a slight displacement of the isotherms towards the cold side (Figure 6).

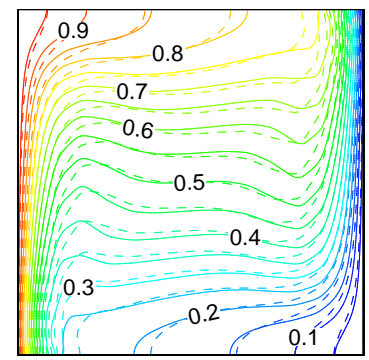

$\mathrm{Ha}=0$

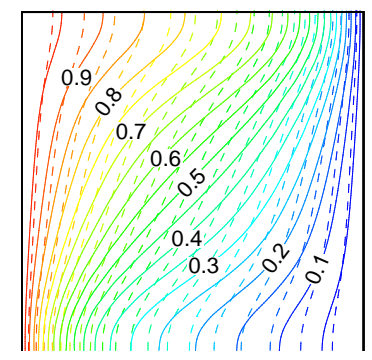

$\mathrm{Ha}=100, \alpha=0^{\circ}$

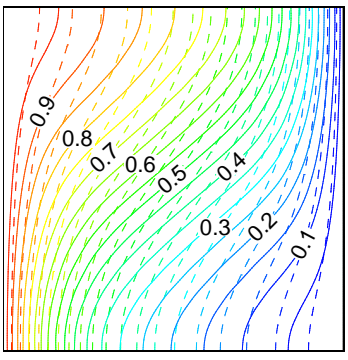

$\mathrm{Ha}=100, \alpha=90^{\circ}$

Figure 6. Isotherms in the mid-plane (solid $\varphi=0.05$, dashed $\varphi=0$ ) for $\operatorname{Ra}=10^{5}$.

Figure 7 presents the effect of increasing CNT volume fraction on the average Nusselt number for various Hartman numbers with considering the effect of the orientation of the magnetic field. 
An enhancement of heat transfer occurs by increasing the nanoparticle volume fraction due to the increase of the thermal conductivity. The effect of the magnetic field opposes the heat transfer and the values of Nusselt number decrease by increasing Hartman values. This effect is more important for $\alpha=90^{\circ}$ except for $\mathrm{Ra}=10^{5}$ at $\mathrm{Ha}=20$ and 40 .

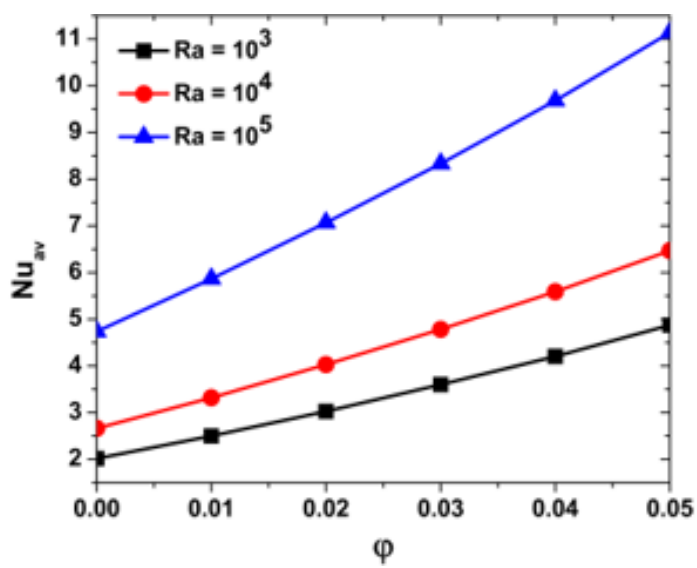

(a) $\mathrm{Ha}=\mathbf{0}$

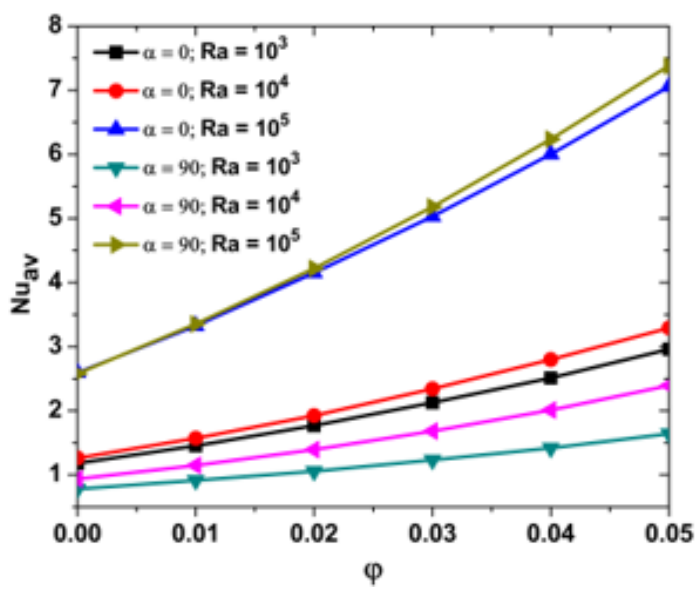

(c) $\mathrm{Ha}=40$

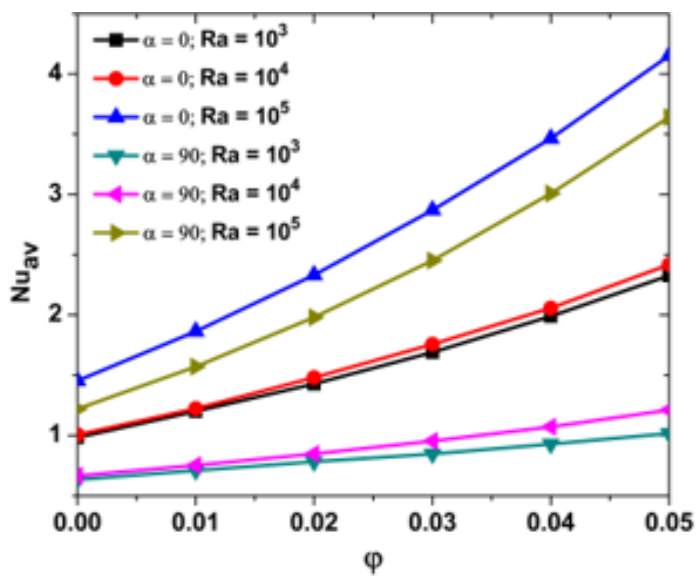

(e) $\mathrm{Ha}=80$

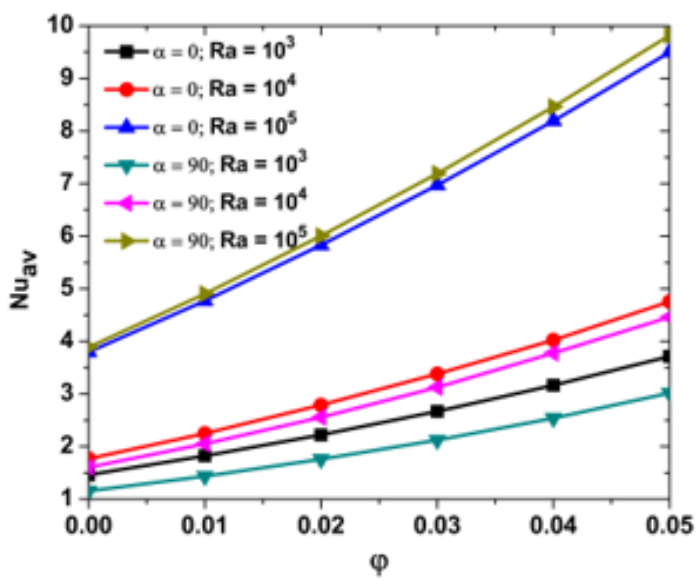

(b) $\mathrm{Ha}=\mathbf{2 0}$

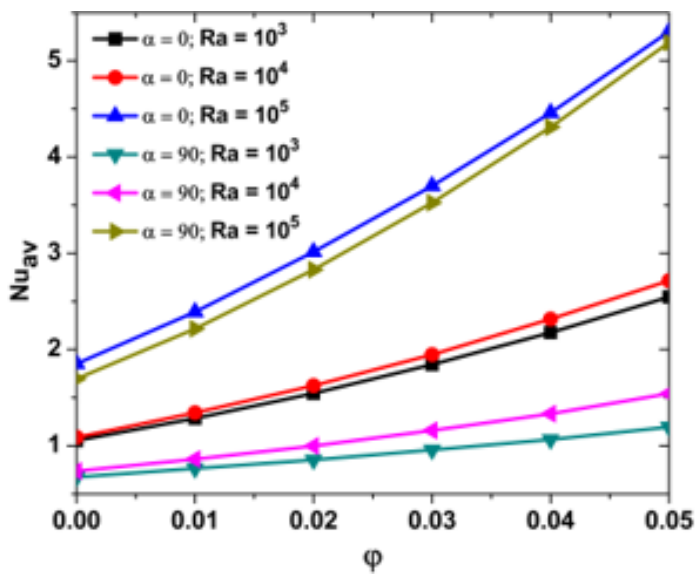

(d) $\mathrm{Ha}=60$

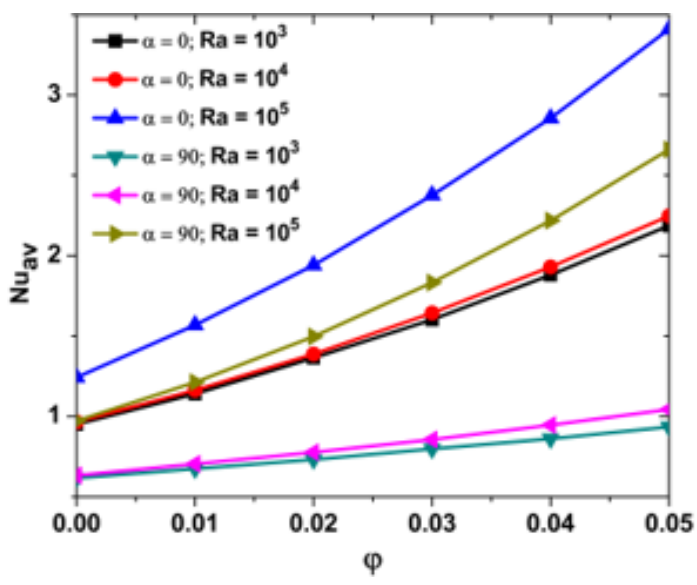

(f) $\mathrm{Ha}=\mathbf{1 0 0}$

Figure 7. Effects of CNT volume fraction and Hartmann number on the mean Nusselt number with $\mathrm{Ma}=1000$ and different Ra number. 
The impacts of the magnetic field direction along with Marangoni number on the mean Nusselt number for different $\mathrm{Ra}, \varphi=0.02$ and $\mathrm{Ha}=40$ are shown on Figure 8 . Obtained results reveal that the rate of heat transfer varies according to the magnetic field inclination particularly at low Rayleigh number values. Similar results have been observed by Purusothaman et al. [30]. For low values of Rayleigh number $\left(\mathrm{Ra}=10^{3}\right)$, the magnetic influence is intensified, and enormous deviations have been noticed between the base fluid and the nanofluid. However, simulations show that the magnetic field direction has insignificant impact on the transferred thermal energy for small Rayleigh number.

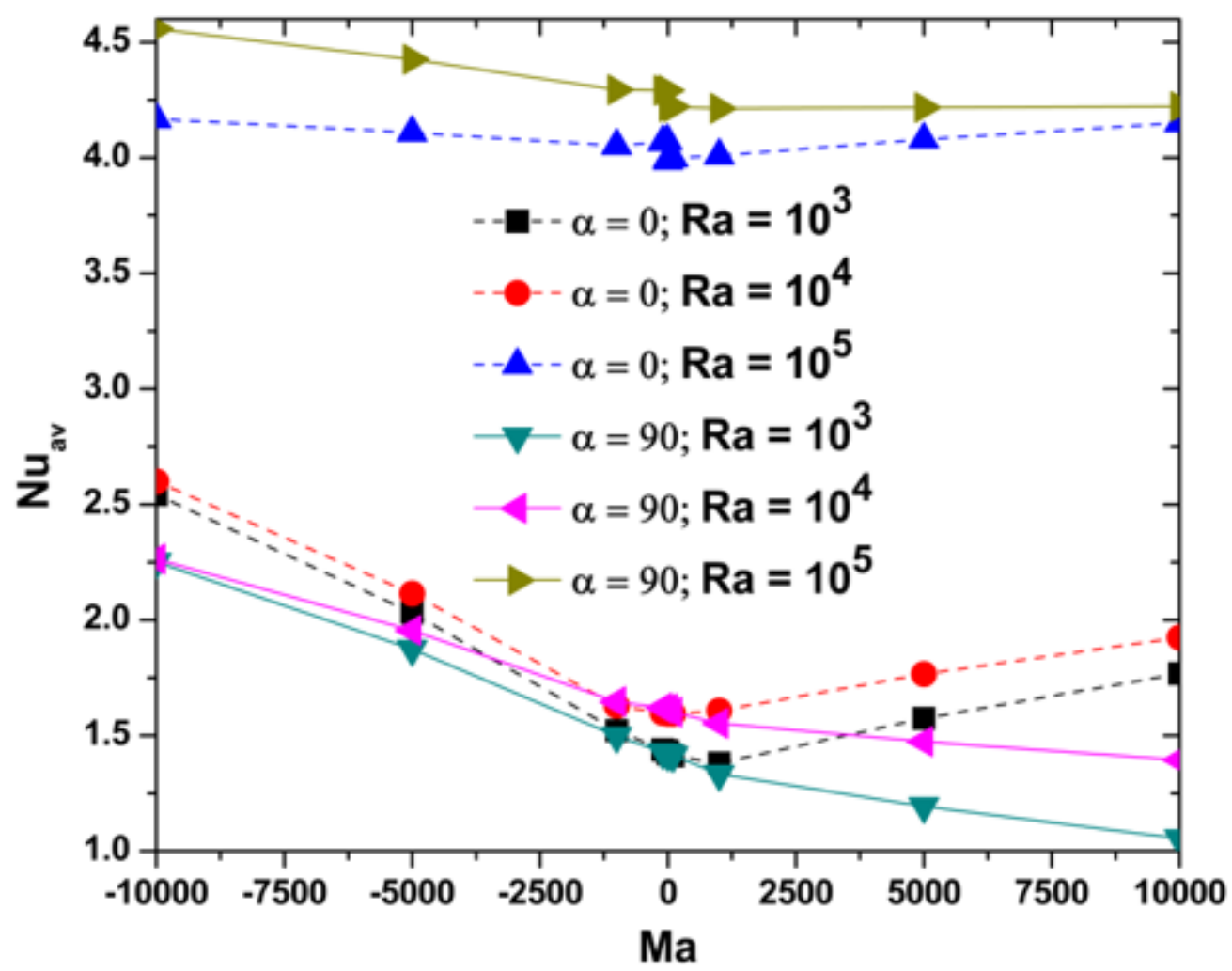

Figure 8. Marangoni number effect on average Nusselt number for different $\mathrm{Ra}, \mathrm{Ha}=40$ and $\varphi=0.02$. $\alpha=0^{\circ}$ (dashed lines) and $\alpha=90^{\circ}$ (solid lines).

Figure 8 depicts the effect of Marangoni number on heat transfer for various Ra values and magnetic field direction. For a vertical magnetic field $\left(\alpha=0^{\circ}\right)$, Nusselt number is decreasing despite the sign of Marangoni number. For a horizontal magnetic field, a minimum exists at $\mathrm{Ma}=10$ and average Nusselt number is decreasing for negative values of Ma and is increasing for positive values. This can be explained by the intensification of the flow that occurs for higher positive Ma values and the incapability of the horizontal magnetic field in opposing efficiently the capillary force as can be deduced from Figure 4 where the capillary induced vortex doesn't disappear compared to the horizontal magnetic field case. This result indicates that the vertical magnetic field is more effective in opposing the capillary and buoyancy forces.

\section{Conclusions}

The current study offers a 3D computational analysis of magnetic field effects on the rate of heat transfer during laminar-thermocapillary free convection coupling in a cavity filled with CNT-water nanofluid. It is worthwhile to mention that no previous works are available to relate the combination of CNT thermocapillary 3D natural convection in the presence of a horizontal or a vertical magnetic field. The effect of adding CNT is investigated and the volume fraction is limited to 0.05 to avoid the changes of the Newtonian behavior of the fluid. It is found that the induced Lorentz forces 
oppose the thermocapillary force and contribute in the control of the flow and in the reduction of heat transfer. The vertical magnetic field is found to be more effective especially for higher Hartman number values. In opposition to magnetic effect the presence of CNT increases the heat transfer rate due to the enhancement of the thermophysical properties.

Author Contributions: In this paper, L.K., S.A. and H.A.M. conceived and designed the numerical Code. L.K., W.H. and E.L. performed the numerical executions. H.A.H., M.A.A. and W.A. analyzed the data. S.A., W.A. and L.K. wrote the paper. All authors have read and agreed to the published version of the manuscript.

Acknowledgments: The authors would like to acknowledge the support provided by the Deanship of Scientific Research at King Khalid University for funding this work through General Research Project under grant number (R.G.P1./95/40).

Conflicts of Interest: The authors declare no conflicts of interest.

\section{Nomenclature}

\begin{tabular}{|c|c|}
\hline$\vec{B}$ & Magnetic field $\left(\overrightarrow{B^{\prime}} / B_{0}\right)$ \\
\hline $\mathrm{C}_{\mathrm{p}}$ & Specific heat at constant pressure $(\mathrm{J} / \mathrm{kg} \mathrm{K})$ \\
\hline$\vec{E}$ & Dimensionless electric field \\
\hline$\vec{e}_{B}$ & Direction of magnetic field \\
\hline$g$ & Gravitational acceleration $\left(\mathrm{m} / \mathrm{s}^{2}\right)$ \\
\hline $\mathrm{Ha}$ & Hartmann number \\
\hline$\vec{J}$ & Dimensionless density of electrical current \\
\hline$k$ & Thermal conductivity (W/m K) \\
\hline$l$ & Enclosure width \\
\hline$M a$ & Marangoni number \\
\hline$n$ & Unit vector normal to the wall \\
\hline$N u$ & Local Nusselt number \\
\hline $\operatorname{Pr}$ & Prandtl number \\
\hline$R a$ & Rayleigh number \\
\hline$t$ & Dimensionless time $\left(t^{\prime} \cdot \alpha / l^{2}\right)$ \\
\hline$\theta$ & Dimensionless temperature $\left[\left(T^{\prime}-T_{c}^{\prime}\right) /\left(T_{h}^{\prime}-T_{c}^{\prime}\right)\right]$ \\
\hline$T_{c}^{\prime}$ & Cold temperature $(\mathrm{K})$ \\
\hline$T_{h}^{\prime}$ & Hot temperature $(\mathrm{K})$ \\
\hline $\mathrm{T}_{\mathrm{O}}$ & Bulk temperature $\left[T_{o}=\left(T^{\prime}{ }_{c}+T^{\prime}{ }_{h}\right) / 2\right](\mathrm{K})$ \\
\hline$\vec{V}$ & Dimensionless velocity vector $\left(\overrightarrow{V^{\prime}} \cdot l / \alpha\right)$ \\
\hline$x, y, z$ & Dimensionless Cartesian coordinates $\left(x^{\prime} / l, y^{\prime} / l, z^{\prime} / l\right)$ \\
\hline \multicolumn{2}{|c|}{ Greek symbols } \\
\hline$\alpha$ & magnetic field inclination \\
\hline$\beta$ & Thermal expansion coefficient $(1 / K)$ \\
\hline$\rho$ & Density $\left(\mathrm{kg} / \mathrm{m}^{3}\right)$ \\
\hline$\mu$ & Dynamic viscosity (kg/m s) \\
\hline$v$ & Kinematic viscosity $\left(\mathrm{m}^{2} / \mathrm{s}\right)$ \\
\hline$\Phi$ & Dimensionless electrical potential \\
\hline$\varphi$ & Nanoparticle volume fraction \\
\hline$\ominus$ & Dimensionless Temperature \\
\hline$\sigma$ & Surface tension \\
\hline$\sigma_{e}$ & Electrical conductivity \\
\hline$\vec{\Psi}$ & Dimensionless vector potential $\left(\overrightarrow{\Psi^{\prime}} / \alpha\right)$ \\
\hline$\vec{w}$ & Dimensionless vorticity $\left(w^{\prime} \cdot \alpha / l^{2}\right)$ \\
\hline$\Delta T$ & Dimensionless temperature difference \\
\hline
\end{tabular}




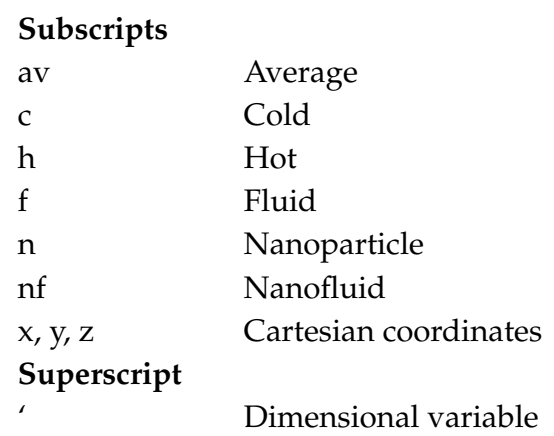

\section{References}

1. Gillon, P.; Homsy, G.M. Combined thermocapillary-buoyancy convection in a cavity: An experimental study. Phys. Fluids 1996, 8, 2953-2963. [CrossRef]

2. Kanouff, M.; Greif, R. Oscillations in thermocapillary convection in a square cavity. Int. J. Heat Mass Transf. 1994, 37, 885-892. [CrossRef]

3. Chen, J.; Hwu, F.S. Oscillatory Thermocapillary Flow in a Rectangular Cavity. Int. J. Heat Mass Transf. 1993, 36, 3743-3749. [CrossRef]

4. Semma, E.; EL Ganaoui, M.; Bennar, R.; Cheddadi, A. Effet de la Convection Thermocapillaire sur les Instabilités de la Phase Fluide et de l'Interaction solide/liquide en Croissance Dirigée. In Proceedings of the 16ème Congrès Français de Mécanique, Dourdan, France, 17-21 September 2018.

5. Behniat, M.; Stella, F.; Gui, G. A Numerical Study of Three-Dimensional Combined Buoyancy and Thermocapillary Convection. Int. J. Multiph. Flow 1995, 21, 529-542. [CrossRef]

6. Babu, V.; Korpela, S.A. Three Dimensional Thermocapillary Convection in a Cavity. Comput. Fluids 1990, 18, 229-238. [CrossRef]

7. Zebib, A.; Homsy, G.M.; Meiburg, E. High Marangoni number convection in a square cavity. Phys. Fluids 1985, 28, 3467-3476. [CrossRef]

8. Ramanan, N.; Korpela, S.A. Thermocapillary convection in an axi-symmetric pool. Comput. Fluids 1990, 18, 205-215. [CrossRef]

9. Morthland, T.E.; Walker, J.S. Convective Heat transfer due to Thermocapillary Convection with a Strong Magnetic Field Parallel to The Free Surface. Int. J. Heat Mass Transf. 1997, 40, 3283-3291. [CrossRef]

10. Rudraiah, N.; Cholappa, V.; Subbaraya, C.K. Combined Surface Tension and Buoyancy-Driven Convection in Rectangular Open Cavity in Presence of a Magnetic Field. Int. J. Non Linear Mech. 1995, 30, 759-770. [CrossRef]

11. Jiang, Y.; Zhou, X.; Wang, Y. Comprehensive heat transfer performance analysis of nanofluid mixed forced and thermocapillary convection around a gas bubble in minichannel. Int. Commun. Heat Mass Transf. 2020, 110, 104386. [CrossRef]

12. Jiang, Y.; Zhou, X. Heat transfer and entropy generation analysis of nanofluids thermocapillary convection around a bubble in a cavity. Int. Commun. Heat Mass Transf. 2019, 105, 37-45. [CrossRef]

13. Kolsi, L.; Lajnef, E.; Aich, W.; Alghamdi, A.; Aichouni, M.A.; Borjini, M.N.; Ben Aissia, H. Numerical investigation of combined buoyancy-thermocapillary convection and entropy generation in 3D cavity filled with $\mathrm{Al}_{2} \mathrm{O}_{3}$ nanofluid. Alex. Eng. J. 2017, 56, 71-79. [CrossRef]

14. Aminfar, H.; Mohammadpourfard, M.; Mohseni, F. Numerical investigation of thermocapillary and buoyancy driven convection of nanofluids in a floating zone. Int. J. Mech. Sci. 2012, 65, 147-156. [CrossRef]

15. Abdullah, A.A.; Alraiqib, N.M.; Lindsay, K.A. Modelling the stability of Marangoni convection in a layer of nanofluid. Int. J. Therm. Sci. 2020, 151, 106228. [CrossRef]

16. Jiang, Y.; Zhou, X. Analysis of flow and heat transfer characteristics of nanofluids surface tension driven convection in a rectangular cavity. Int. J. Mech. Sci. 2019, 153-154, 154-163. [CrossRef]

17. Zhuang, Y.J.; Zhu, Q.Y. Numerical study on combined buoyancy-Marangoni convection heat and mass transfer of power-law nanofluids in a cubic cavity filled with a heterogeneous porous medium. Int. J. Heat Fluid Flow 2018, 71, 39-54. [CrossRef] 
18. Lee, K.J.; Kamotani, Y.; Yoda, S. Combined thermocapillary and natural convection in rectangular containers with localized heating. Int. J. Heat Mass Transf. 2002, 45, 4621-4630. [CrossRef]

19. Sheikholeslami, M.; Chamkha, A.J. Influence of Lorentz forces on nanofluid forced convection considering Marangoni convection. J. Mol. Liq. 2017, 225, 750-757. [CrossRef]

20. Estellé, P.; Cabaleiro, D.; Żyła, G.; Lugo, L.; Murshed, S.M.S. Current trends in surface tension and wetting behavior of nanofluids. Renew. Sustain. Energy Rev. 2018, 94, 931-944. [CrossRef]

21. Zhuang, Y.J.; Zhu, Q.Y. Analysis of entropy generation in combined buoyancy-Marangoni convection of power-law nanofluids in 3D heterogeneous porous media. Int. J. Heat Mass. Transf. 2018, 118, 686-707. [CrossRef]

22. Huang, H.L.; Zhang, Y.; Zhu, G.P. Thermocapillary flow and free-surface deformation of liquid bridge under different magnetic fields. Appl. Therm. Eng. 2018, 135, 83-94. [CrossRef]

23. Yu, J.J.; Li, Y.R.; Ruan, D.F.; Wu, C.M. Aspect ratio and capillary ratio dependence of thermal-solutal capillary-buoyancy flow of a binary mixture in an annular pool. Int. J. Therm. Sci. 2019, 136, 347-356. [CrossRef]

24. Saleh, H.; Hashim, I. Buoyant Marangoni convection of nanofluids in square cavity. Appl. Math. Mech. 2015, 36, 1169-1184. [CrossRef]

25. Jiang, Y.N.; Zhou, X.M. Numerical study of heat transfer and entropy generation of nanofluids buoyant-thermocapillary convection around a gas bubble. Microgravity Sci. Technol. 2019, 31, 195-206. [CrossRef]

26. Mahmoudi, A.H.; Pop, I.; Shahi, M. Effect of magnetic field on natural convection in a triangular enclosure filled with nanofluid. Int. J. Therm. Sci. 2012, 59, 126-140. [CrossRef]

27. Ozoe, H.; Okada, K. The Effect of Direction of the External Magnetic Field on the Three-Dimentional Convection in a Cubical Enclosure. Int. J. Heat Mass Transf. 1989, 32, 1939-1954. [CrossRef]

28. Al-Rashed, A.A.A.A.; Kalidasan, K.; Kolsi, L.; Aydi, A.; Malekshah, E.H.; Hussein, A.K.; Kanna, P.R. Three-Dimensional Investigation of the Effects of External Magnetic Field Inclination on Laminar Natural Convection Heat Transfer in CNT-Water Nanofluid Filled Cavity. J. Mol. Liq. 2018, 252, 454-468. [CrossRef]

29. Xue, Q.Z. Model for thermal conductivity of carbon nanotube-Based composites. Phys. B 2005, 368, 302-307. [CrossRef]

30. Purusothamana, A.; Oztop, H.F.; Nithyadevi, N.; Abu-Hamdeh, N.H. 3D natural convection in a cubical cavity with a thermally active heater under the presence of an external magnetic field. Comput. Fluids 2016, 128, 30-40. [CrossRef] 